\title{
Primary hematological malignancy of the uterine cervix: A case report
}

\author{
WEI GUI $^{1 *}$, JING LI $^{2 *}$, ZONG ZHANG ${ }^{1}$, LIEYANG WANG ${ }^{1}$, \\ $\mathrm{JIN} \mathrm{ZHAO}^{1}$, LI MA ${ }^{1}$ and LIPING SU ${ }^{1}$ \\ Departments of ${ }^{1}$ Hematology and ${ }^{2}$ Pathology, Affiliated Shanxi Tumor Hospital \\ of Shanxi Medical University, Taiyuan, Shanxi 030013, P.R. China
}

Received October 21, 2018; Accepted March 1, 2019

DOI: 10.3892/ol.2019.10652

\begin{abstract}
The present case report investigated the clinical characteristics of primary hematological malignancy of the uterine cervixin five patients. Among the five patients, three patients were diagnosed with non-Hodgkin's lymphoma (NHL) and two patients with myeloid sarcoma (MS) of the uterine cervix. Biopsies of the uterine cervices demonstrated the involvement of lymphoid cells or myeloid blasts cells. Immunohistochemical staining demonstrated the expression of B-lymphoid and myeloid lineage markers. The associated lysozyme, myeloperoxidase and mast/stem cell growth factor receptor (CD117) markers were specific for the diagnosis of MS. The three patients with NHL survived, and one of the patients survived for 82 months with no evidence of disease; however, was eventually lost to follow-up. The two patients with MS succumbed to the cancer as a result of progressive disease and leukemia. Therefore, pathological examination may be important for the timely diagnosis and appropriate therapeutic regimen for primary hematological malignancy of the uterine cervix.
\end{abstract}

\section{Introduction}

Primary hematological malignancy of the uterine cervix is rare and thus, the literature regarding the disease is primarily limited to case reports and small case series, to the best of the authors' knowledge (1-5). The uterine cervix was previously identified to be involved in one out of 730 cases of non-Hodgkin's lymphoma (NHL) and 1 out of 175 cases of extra-nodal lymphoma in the UK in 1974 (1). Furthermore, myeloid sarcoma (MS) was observed in $3-5 \%$ of the patients

Correspondence to: Dr Liping Su, Department of Hematology, Affiliated Shanxi Tumor Hospital of Shanxi Medical University, 3 Shanxi Zhigongxinjie Street, Taiyuan, Shanxi 030013, P.R. China

E-mail: suliping_2018@163.com

*Contributed equally

Key words: primary hematological malignancy of the uterine cervix, non-Hodgkin's lymphoma, myeloid sarcoma with acute myeloid leukemia (AML) (5). There are $\sim 0.7$ per 1,000,000 young individuals who are diagnosed with MS and $\sim 2$ per 1,000,000 individuals in the adult population who are diagnosed with MS (5). The most common symptom of MS is vaginal bleeding, thus, the pathological diagnosis of NHL and MS is very important. In the present case report, the pathological reports of three patients with NHL and two patients with MS are described. Immunohistochemical (IHC) staining demonstrated the presence of B-lymphoid in the patients with NHL and the presence of myeloid lineage markers in the patients with MS. The cancer of the patients was managed with chemotherapy and either radiotherapy/surgery alone or together. Hematopoietic stem cell transplantation (HSCT) may improve the prognosis for patients with MS. For example, Chen et al (6) reported that 4 patients with MS underwent allogeneic HSCT (allo-HSCT). Among them, 3 patients remained alive with complete remission (CR) (6).

\section{Case report}

Case 1. A 65-year-old female was admitted to The Department of Obstetrics and Gynecology at The Shanxi Tumor Hospital (Shanxi, China) in January 2016 after 2 weeks of postmenopausal vaginal bleeding. A biopsy of the cervical mass identified the presence of a suspicious uterine cervical cancer. A physical examination demonstrated no evidence of superficial lymph node enlargement or hepatosplenomegaly. A gynecological examination demonstrated that the uterine cervix was $6 \mathrm{~cm}$ in size and featured an endogenous mass; a mass had grown on the inside of the cervical canal, which could not be seen from the outside of the cervix. Laboratory tests demonstrated that the hemoglobin (HGB) count was $140 \mathrm{~g} / 1$, the white blood cell (WBC) count was $6.2 \times 10^{9} / 1$ and the platelet (PLT) count was $3.8 \times 10^{11} / 1$. The lactate dehydrogenase (LDH), $\beta 2$-microglobulin $(\beta 2 \mathrm{MG})$, liver and renal function, coagulation test, Epstein Barr virus (EBV), cytomegalovirus (CMV) and erythrocyte sedimentation rate (ESR) results were all normal. There was no detectable abnormity in the bone marrow (BM) smear, BM biopsy (BMB) or flow cytometry (FCM) analysis of the BM cells. Computerized tomography (CT) demonstrated bilateral inguinal, para-iliac vessels and retroperitoneal lymph node enlargement (maximum lymph node size was $0.8 \mathrm{x} 0.9 \mathrm{~cm}$ ). The patient underwent adnexectomy and resection of pelvic 
lymph nodes. A pathological examination identified diffuse large B cell lymphoma (DLBCL) of the uterine cervix (Fig. 1). The tumor was $6 \times 5 \times 4 \mathrm{~cm}$ and was involved in full-thickness cervical stromal invasion. Chronic endometritis and reactive hyperplasia of 31 pelvic lymph nodes were confirmed. IHC staining demonstrated the presence of cells that were positive for B-lymphocyte antigen CD20 (CD20), Myc proto-oncogene protein (c-Myc), B-cell lymphoma (Bcl)-2, Bcl-6, multiple myeloma oncogene1 (MUM1), tumor protein p63 (p63) and proliferation marker protein Ki-67 (Ki67) 80\%+, and were negative for T-cell surface glycoprotein CD3 $\varepsilon$ chain (CD3), neprilysin (CD10), Fc $\gamma$ RIII (CD16), neural cell adhesion molecule 1 (CD56), cytokeratin (CAM5.2), cytokeratin pan (AE)1/ AE3 and myeloperoxidase (MPO). The patient was diagnosed with primary DLBCL of the uterine cervix stage IIEA, with an International Prognosis Index (IPI) score of 1 and an Eastern Cooperative Oncology Group (ECOG) score of 1 (7). The patient received a regimen of rituximab $(\mathrm{R})$-cyclophosphamide, pirarubicin, vincristine and dexamethasone (R-CHOP) for six cycles. CR was achieved, and R-CHOP was administered for two additional cycles. A follow-up of the patient was conducted after 35 months with no evidence of recurrence.

Case 2. A 43-year-old female was admitted to The Department of Obstetrics and Gynecology at the Shanxi Tumor Hospital in January 2005 with a 1-year history of increased leucorrhea and contact vaginal bleeding. Gynecological and ultrasound examinations identified the presence of a $4.4 \times 5.1 \mathrm{~cm}$ mass of the uterine cervix. The laboratory tests demonstrated that the routine blood, liver and renal function, coagulation test, LDH and $\beta_{2} \mathrm{MG}$ results were all normal. The patient underwent adnexectomy resection of a $4 \mathrm{~cm}$ mass between the uterine cervix and the upper section of the vagina. A pathological examination demonstrated mucosa-associated lymphoid tissue lymphoma (MALToma) of the uterine cervix, endometrial proliferation, reactive hyperplasia of 9 pelvic lymph nodes, and there was no evidence of abnormities in the vaginal stump and ovary. IHC staining demonstrated that the cells were positive for CD20, B-cell antigen receptor complex-associated protein $\beta$ chain and Ki67 10\% ${ }^{+}$, and were negative for CD3, AE1/AE3, muscle creatine kinase, p63, synapsin (Syn), chromogranin A glycoprotein hormones $\alpha$-chain (CGA) and myogenin. The patient was transferred to The Department of Hematology at The Shanxi Tumor Hospital. There was no evidence of superficial lymph node enlargement or hepatosplenomegaly, and a BM smear/BMB did not raise any cause for concern. The patient was diagnosed with primary MALToma of the uterine cervix stage IEA, with an IPI score of 0 and an ECOG score of 1 . The patient received a regimen of $\mathrm{CHOP}$ with etoposide for six cycles and achieved CR. The patient survived 82 months with no evidence of disease (NED) and was lost to follow-up during 2012.

Case 3. A 36-year-old female was admitted to The Department of Hematology at The Shanxi Tumor Hospital in January 2011 with a 1-month history of vaginal bleeding. A gynecological examination identified a mass of $4 \times 3 \mathrm{~cm}$ in the uterine cervix. A biopsy demonstrated DLBCL of the cervical mass and chronic vaginitis of the upper section of the vagina. According to the consultation with the resident pathologists, IHC staining demonstrated that the cells were positive for leukocyte common antigen (LCA), CD20, p63 and $\mathrm{Ki} 6780 \%^{+}$and were negative for CD3, CD56, casein kinase II subunit $\alpha$, MPO, epithelial membrane antigen (EMA), CGA, Syn and human melanoma black 45. The pathologists agreed with the DLBCL diagnosis. During the physical examination, no superficial lymph node enlargement or hepatosplenomegaly was observed. The laboratory tests demonstrated that routine blood $\mathrm{LDH}, \beta_{2} \mathrm{MG}$, liver and renal function, EBV, CMV and ESR were normal. A BM smear/BMB did not raise any cause for concern. An ultrasound examination identified a small myoma of the uterus. The patient was diagnosed with primary DLBCL of the uterine cervix stage IEA with an IPI score of 0 and an ECOG score of 1 . The patient received a regimen of $\mathrm{CHOP}$ for six cycles and achieved CR. The patient survived for 73 months with NED.

Case 4. A 46-year-old female received a routine health examination. The gynecological examination identified a mass of $6 \times 5 \mathrm{~cm}$ of the uterine cervix. The left upper section of the vaginal wall was possibly involved. The uterus was $7 \times 5 \mathrm{~cm}$ and left parametric narrow. The patient was admitted to The Department of Obstetrics and Gynecology at The Shanxi Tumor Hospital in December 2012. A physical examination did not identify any superficial lymph node enlargement or hepatosplenomegaly. Laboratory tests demonstrated that HGB $133 \mathrm{~g} / \mathrm{l}$, WBC $7.71 \times 10^{9} / 1$, PLT $1.62 \times 10^{11} / 1, \mathrm{LDH}, \beta_{2} \mathrm{MG}$, liver and renal function, coagulation test, EBV, CMV and ESR were all normal. A BM smear/BMB did not raise any cause for concern. FCM of BM did not demonstrate any abnormal expression levels of CD10, CD14 or CD64. An ultrasound examination identified a mass of $6 \times 5 \mathrm{~cm}$ of the uterine cervix and normal uterus. A biopsy of the mass in the uterine cervix confirmed the diagnosis of MS. IHC staining demonstrated that cells were positive for LCA, MPO, CD99 antigen-like protein 2 (CD99), CD117, lysozyme and $\mathrm{Ki} 67$ 70\%+, and were negative for CD3, T-cell surface glycoprotein CD5, CD10, Sialyl-Lewis X (CD15), CD20, FceRII, tumor necrosis factor receptor superfamily member 8 , hematopoietic progenitor cell antigen CD34 (CD34), ADP-ribosyl cyclase/cyclic ADP-ribose hydrolase 1 (CD38), macrosialin, anaplastic lymphoma receptor tyrosine kinase receptor (ALK), ALK tyrosine kinase receptor, Bcl-6, cell cycle protein D1, ubiquitin-conjugating enzyme E2 D1, MUM1, myogenin, desmin, paired box protein Pax-5 and terminal deoxynucleotide transferase. DNA nucleotidylexotransferase. Histological analysis of the cervical mass at The Beijing Friendship Hospital (Beijing, China) demonstrated that the neoplastic cells were medium in size with chromatin, with morphology and IHC features typically associated with blasts, and the diagnosis confirmed MS. The clinical diagnosis was primary MS of the uterine cervix stage IIEA, with an IPI score of 0 and ECOG score of 1 . At the request of the patient, adnexectomy was performed. The uterine cervix was not resected due to involvement of the left wall of the upper section of the vagina. A pathological examination identified a myoma in the uterus. After 3 weeks, the patient complained of a yellow fluid coming from the vagina. Consultation with an urologist confirmed ureteral vaginal fistulas, whereas, a CT scan demonstrated left pyeloureterectasis. Mild left hydronephrosis suggested that the patient required surgery. The surgeon identified a severe lower ureteral stricture and adhesion near the bladder. Left ureterocystostomy and catheterization of the 
Table I. Clinical data for primary hematological malignancy of uterine cervix.

\begin{tabular}{|c|c|c|c|c|c|c|c|c|c|}
\hline $\begin{array}{l}\text { Case } \\
\text { number }\end{array}$ & $\begin{array}{l}\text { Age, } \\
\text { years }\end{array}$ & LDH, U/1 & Stage & $\begin{array}{l}\text { Mass of uterine } \\
\text { cervix, cm }\end{array}$ & Biopsy & $\begin{array}{l}\text { Pathological } \\
\text { type }\end{array}$ & Treatment & $\begin{array}{l}\text { Treatment } \\
\text { outcome }\end{array}$ & $\begin{array}{l}\text { OS, } \\
\text { months }\end{array}$ \\
\hline 1 & 65 & 221 & IIEA & $6 \times 2$ & Yes & DLBCL & Surgery, CT & Alive with $\mathrm{CR}$ & 35 \\
\hline 2 & 43 & 210 & IEA & 4.5 & No & MALToma & Surgery, CT & Alive with CR & $82^{\mathrm{a}}$ \\
\hline 3 & 36 & 169 & IEA & $4 \times 3$ & Yes & DLBCL & $\mathrm{CT}$ & Alive with CR & 73 \\
\hline 4 & 46 & 133 & IIEA & $6 \times 5$ & Yes & MS & Surgery, CT & $\begin{array}{l}\text { Succumbed to } \\
\text { progressive disease }\end{array}$ & 21 \\
\hline 5 & 39 & 1,014 & IVEA & $6.9 \times 5.6$ & Yes & MS & Surgery, CT & $\begin{array}{l}\text { Succumbed to } \\
\text { leukemia }\end{array}$ & 26 \\
\hline
\end{tabular}

${ }^{\text {aL }}$ ost to follow-up. MALToma, mucosa associated lymphoid tissue lymphoma; DLBCL, diffuse large B cell lymphoma; MS, myeloid sarcoma; $\mathrm{CT}$, chemotherapy; CR, complete remission; $\mathrm{LDH}$, lactate dehydrogenase; OS, overall survival.
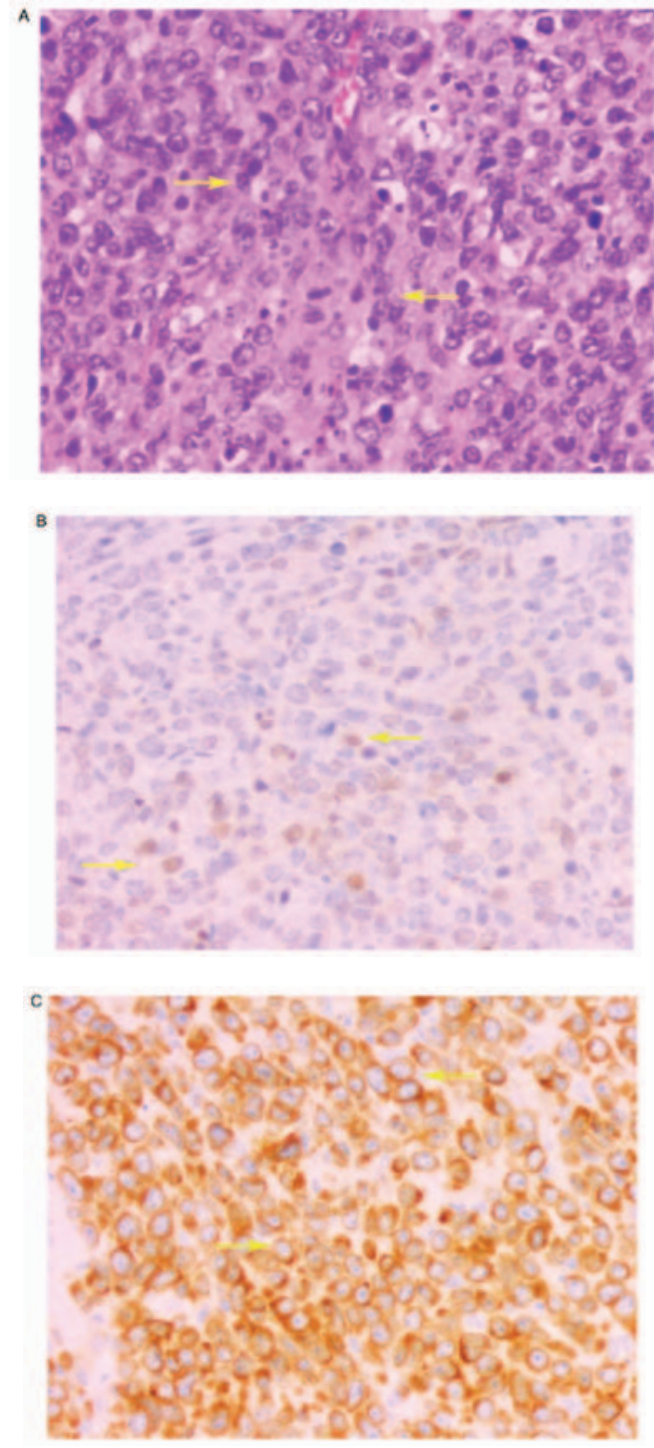

Figure 1. Case 1. (A) Histological staining of the uterine cervix demonstrating diffuse infiltration of lymphoid cells. These cells were seen in the interstitial tissues. The nucleus was deeply stained with hematoxylin and eosin, and the number of nucleoli was 1-2. Lymphoid tumor cells are indicated (yellow arrows). The mitotic figures were visible. Magnification, x200. Immunohistochemical staining demonstrating positive expression of (B) proto-oncogene protein (cMyc) in neoplastic cells (yellow arrows). The nucleus was deeply stained and (C) neoplastic cells expressed BCL-2 protein (yellow arrows), with a deeply stained cell membrane. Magnification, x200. left ureter with a D-J tube were performed. The patient was transferred to The Department of Hematology at The Shanxi Tumor Hospital subsequent to pulling out the D-J tube. A BM smear did not raise any cause for concern. A CT scan identified the presence of a $4.4 \times 3.2 \mathrm{~cm}$ mass in the right renal outer edge; right iliac region lymph nodes (maximum lymph node size was $1.2 \mathrm{~cm}$ ) and left pyeloureterectasis. The patient received a regimen of idarubicin and arabinoside (Ara-c) (IA) for three cycles, and a regimen of aclacinomycin and Ara-c for two cycles. An ultrasound examination identified mild bilateral hydronephrosis and right upper ureterectasia. The patient achieved partial remission. The patient was administered IA for three cycles and homoharringtonine and Ara-c for one cycle, and achieved CR. After 3 months, an ultrasound examination identified bilateral cervical, inguinal and para-iliac vessels, lymph node enlargement, bilateral hydronephrosis and bilateral ureterectasia. A regimen of mitoxantrone and Ara-c (MA) was administered for two cycles. An ultrasound examination identified the presence of a $4.2 \times 4.5 \mathrm{~cm}$ mass in the right pelvis with bilateral hydronephrosis. The BM smear was normal. The patient succumbed to the cancer 21 months after diagnosis.

Case 5. A 39-year-old female was admitted to The Department of Obstetrics and Gynecology at The Shanxi Tumor Hospital in February 2014 with a 5-day history of vaginal bleeding. Gynecological examination identified a $6.9 \times 5.4 \mathrm{~cm}$ mass of the uterine cervix. An ultrasound examination identified a mass in the uterine cervix and involvement of the lower section of the uterus. A biopsy identified medium size neoplastic cells. IHC staining demonstrated that the cells were positive for LCA, MPO, CD34, CD38, leukosialin (CD43), CD56, CD99, CD117, lysozyme and $\mathrm{Ki} 6780 \%^{+}$, and were negative for $\mathrm{CD} 3$, T cell antigen $\mathrm{CD} 7$, cluster of differentiation 8, neprilysin (CD10), CD15, CD20, AE1/AE3, CGA, Syn, desmin and vimentin. The pathological diagnosis confirmed MS of the uterine cervix. Positron emission tomography-computed tomography (PET-CT) demonstrated bilateral cervical, near the abdominal aorta, right para-iliac vessel large lymph nodes, and identified a $6.1 \times 3.7-\mathrm{cm}$ mass in the uterine cervix in the lower part of the uterus. The standard uptake value maximum was 6.79 and 4.63 for the uterine cervix and BM, respectively. The laboratory test demonstrated counts of HGB $67 \mathrm{~g} / 1$, WBC $31.3 \times 10^{9} / 1$, PLT $5.9 \times 10^{10} / 1$ and LDH $1,014 \mathrm{U} / 1$. BM and a peripheral blood 

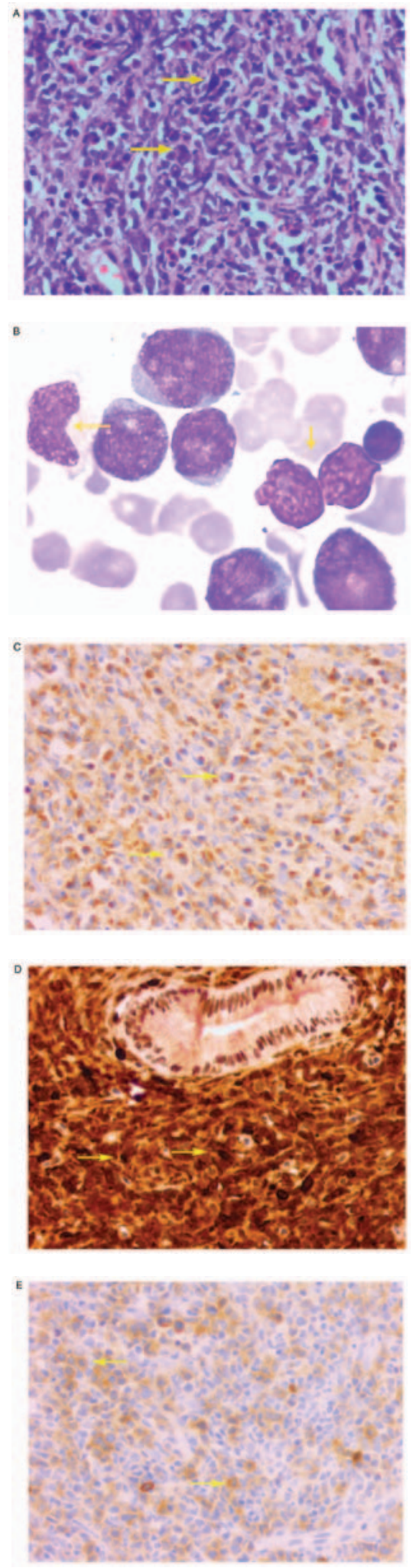

Figure 2. Case 5. (A) Histological staining of the uterine cervix demonstrating neoplastic cells (yellow arrows) were scattered with lymphocytes and inflammatory cells. The nucleus was deeply stained with hematoxylin and eosin, and the nucleolus was not obvious. Magnification, x200. (B) Bone marrow smear demonstrating myeloblasts (yellow arrows) with large nuclei, deep chromatin and coarse particles. Magnification, x1,000. (C) Immunohistochemical staining demonstrated neoplastic cells (yellow arrows) with positive expression of myeloperoxidase (MPO) in the cytoplasm, with scattered granular matter. (D) Neoplastic cells had more dark-stained lysozymes (yellow arrows), and the cytoplasm was positive for lysozyme. (E) Neoplastic cells expressed mast/stem cell growth factor receptor (CD117) (yellow arrows), and the cytoplasm and membrane were positive for CD117. Magnification, x200. smear demonstrated the presence of myeloblasts at 16 and $11 \%$, respectively (Fig. 2). A BMB presented a cluster distribution of myeloblasts. FCM of a BM smear demonstrated positive expression of integrin $\alpha \mathrm{M}$, membrane alanyl aminopeptidase, CD15, CD16, sialic acid binding Ig-like lectin 3, CD34, CD117 and human leukocyte antigen-DR isotype, and was negative for CD10. The patient was diagnosed with MS of the uterine cervix stage IVEA. The patient received an IA regimen for four cycles and an MA regimen for two cycles, and achieved CR. After 5 months, the patient complained of hemorrhagic vaginal secretions. A BM smear demonstrated blasts at $21.5 \%$ and AML was confirmed. The patient received a regimen of IA and CIG regimen and (Ara-c, idarubicin and human granulocyte colony stimulating factor) for two cycles each. A BM smear, performed approximately 2 months later, demonstrated blasts at $2 \%$, which was a lower percentage than in the first BM smear. The patient achieved CR. At the request of the patient, adnexectomy was performed. A pathological diagnosis later demonstrated chronic cervicitis, cervical erosion and endometrial proliferation. After 6 months, the patient reported vaginal hemorrhagic secretions discharges again. An ultrasound examination demonstrated a mass in the vaginal stump. A BM smear identified myeloblasts at $63.6 \%$. The patient succumbed to the cancer 26 months after the initial diagnosis (Table I).

\section{Discussion}

NHL affects extra-nodal sites in one-third of primary hematological malignancies of the uterine cervix, and the majority of the extra-nodal sites are present in the gastrointestinal tract. Primary extra-nodal NHL of the uterine cervix is extremely rare. The uterine cervix was previously identified to be involved in one out of 730 cases of NHL and one out of 175 cases of extra-nodal lymphoma (1). Primary MS of the uterine cervix is additionally uncommon. These diseases were only reported in the form of case reports and small case series (2-4). MS is present in 3-5\% of patients with AML and represents a disease which is encountered in $\sim 0.7$ young individuals per 1,000,000 and 2 per 1,000,000 in the adult population (5). MS may occur de novo, concurrently with AML or as blastic transformation of myelodysplastic syndrome. Antecedent myeloid neoplasms or concurrent AML is present in approximately two-thirds of the cases. It was previously demonstrated that in 21 cases of MS, four cases involved lesions in the uterine cervix and three of these patients were diagnosed with antecedent AML $(6,8)$. Therefore, MS of the uterine cervix was secondary to AML. Frequent sites of MS include the skin, lymph nodes, mediastinum, gastrointestinal tract, bones, brain, ovary, uterine cervix and testis $(3,6)$.

The diagnosis of primary lymphoma of the uterine cervix was determined by pathological evaluation; the disease was additionally localized to the uterine cervix without evidence of involvement of other organs or lymph nodes. Cases 1-4 were confirmed to possess a primary malignancy. Case 5 was diagnosed with an advanced stage cancer with BM involvement; $\mathrm{BM}$ and a peripheral blood smear demonstrated the presence of myeloblasts was $<20 \%$ and therefore, AML was not diagnosed and primary MS was considered. The majority of cervical lymphomas, as determined by histological examination, are DLBCL with diffuse infiltration of atypically large lymphoid cells (1). IHC staining demonstrated that cells were positive 
for LCA, CD20, Bcl-2, Bcl-6 and MUM1. IHC staining of the patient in case 1 demonstrated that cells were positive for c-Myc and Bcl-2. The prognosis for cases involving the co-expression of these two proteins is poor (9). Therefore, case 1 received $\mathrm{R}$ in addition to the $\mathrm{CHOP}$ regimen and achieved $\mathrm{CR}$, although long-term follow-up is necessary. Histology of MS demonstrated neoplastic cells were medium sized and consistently had a blast-like morphology. IHC staining demonstrated positive cells for LCA, CD34, CD38, CD43, CD117, MPO and lysozyme. Positive expression of MPO, lysozyme and CD117 confirmed the diagnosis of MS. Imaging modalities, including ultrasound, magnetic resonance imaging and PET-CT, are useful in the detection of lymphoma in the uterine cervix. PET-CT may be used for detecting early foci, analyzing the extent of the disease and assessing the treatment response $(10,11)$.

The majority of primary cervical NHL cases are either stage I or II. In such cases, the prognosis is considered to be good, with an overall 5-year survival rate of 77\%. These cases were managed with pelvic radiotherapy or neoadjuvant chemotherapy, successfully applied as a first line of treatment (2) Mouhajir et al (2) identified a case of primary NHL of the uterine cervix treated with CHOP and external radiotherapy. The patient achieved CR and survived 16 years with NED. According to the previous study, chemotherapy based on $\mathrm{CHOP}$ alongside moderate doses of radiation was deemed to be the most successful mode of treatment (2). Similarly, in the present study, case 3 achieved a favorable outcome from $\mathrm{CHOP}$ chemotherapy alone. The addition of $\mathrm{R}$ may improve progression-free survival (12). Case 1 received R-CHOP for eight cycles and the effect of this treatment was satisfactory. The prognosis of patients with cervical MS appears to be poor, with $<20 \%$ of cases surviving (4). MS is a highly aggressive type and with a fulminant course. At present, chemotherapy is the main regimen for AML and MS. Cases were treated with a standard induction regimen for AML and achieved CR, although they eventually succumbed to the progressive disease. However, prognosis may be improved if such individuals were to undergo allo-HSCT. Recently, a 40-year-old female patient with MS of the larynx who additionally presented with AML-M2 was treated at The Shanxi Tumor Hospital. Following CR, the patient underwent allo-HSCT. At the time of writing, 36 months had passed since transplantation and the patient continues to experience $\mathrm{CR}$. In previous unpublished data, Chen et al (6) identified three cases of ovarian and vulvae MS with AML. Allo-HSCT improved the prognosis of these patients. Therefore, allo-HSCT requires consideration for patients with MS following CR.

\section{Acknowledgements}

Not applicable.

\section{Funding}

No funding was received.

\section{Availability of data and materials}

The datasets used and/or analyzed during the present study are available from the author upon reasonable request.

\section{Authors' contributions}

WG collected and analyzed the clinical data, reviewed the literature, designed the study, drafted the work and revised it critically for important intellectual content. JL was responsible for the pathological diagnosis. $\mathrm{ZZ}$ analyzed the results of the bone marrow smear. LW, JZ and LM participated in the collection of clinical data. LS participated in the design of the study. All authors read and approved the final manuscript.

\section{Ethics approval and consent to participate}

Not applicable.

\section{Patient consent for publication}

Informed consent was obtained from the patients and their families for the publication of this data.

\section{Competing interests}

The authors declare that they have no competing interests.

\section{References}

1. Sharma V, Dora T, Patel M, Sancheti S and Sridhar E: Case report of diffuse large $B$ cell lymphcma of uterine cervix treated at a Semiurban Cancer Center in North India. Case Rep Hematol 2016: 3042531, 2016.

2. Mouhajir N, Diakité A, Toulba A, Hemmich M, Saadi I, Elkacemi H, Kebdani T and Benjaafar N: Primary non-hodgkin lymphoma of the uterine cervix: case report of long-term survival patient. J Obstet Gynaecol India 64 (Suppl 1): S145-S147, 2014.

3. Parnis J, Camilleri DJ, Babic D, DeGaetano J and Savona-Ventura C: Lymphoma of the cervix. Case Rep Hematol 2012: 326127, 2012.

4. Gill H, Loong F, Mark V, Chan K, Au WY and Kwong YL: Myeloid sarcoma of the uterine cervix presenting as missed abontion. Arch Grnecol Obstet 286: 1339-1341, 2012.

5. Gunyeli I, Kose SA, Ozkaya O, Kose AG, Karabulut A and Kapucuoglu N: Granulocytic sarcoma of the cervix: Is hysterectomy necessary? J Obstet Gynaecol 35: 315-316, 2015.

6. Chen DB, Zhang H, Zhang YH, Wang Y, Song QJ, Yang SM, Cui H, Zhao Y, Fang XZ and Shen DH: Analysis of proliferative lesions of haematopoietic and lymphoid tissue in the female productive tract. Zhonghua Fu Chan Ke Za Zhi 53: 263-269, 2018 (In Chinese).

7. Sehn LH, Berry B, Chhanabhai M, Fitzgerald C, Gill K, Hoskins P, Klasa R, Savage KJ, Shenkier T, Sutherland J, et al: The revised International Prognostic Index (R-IPI) is a better predictor of outcome than the standard IPI for patients with diffuse large B-cell lymphoma treated with R-CHOP. Blood 109: 1857-1861, 2007.

8. Jiang YJ, Wang HX, Zhuang WC, Chen H, Zhang C, Li XM, Zhu GH and He Y: Clinical and pathologic features of myeloid sarcoma. Zhongguo Shi Yan Xue Ye Xue Za Zhi 25: 926-931, 2017 (In Chinese).

9. Yu WJ, Cao LH, Wang JH, Wang ZM, Qian WB, Tong HY, Meng HT, Mai WY, Mao LP, Qian JJ and Jin J: Prognostic significance of proteins expression by immunohistochemical method in diffuse large B cell lymphoma. Zhonghua Xue Ye Xue Za Zhi 38: 784-788, 2017 (In Chinese).

10. Zheng LC, OuYang XL, Zhang WJ, Liu GC and Zhang XM: 18F-FDG PET/CT of primary cervical granulocytic sarcoma. Clin Nucl Med 40: 917-918, 2015.

11. Korivi BR, Jensen CT, Patnana M, Patel KP and Bathala TK: A rare presentation of lymphoma of the cervix with cross-sectional imaging correlation. Case Rep Radiol 2014: 157268, 2014.

12. Baijal G, Vadiraja BM, Fernandles DJ and Vidyasagar MS: Diffuse large B-cell lymphoma of the uterine cervix: A rare case managed novelly. J Cancer Res Ther 5: 140-142, 2009. 